\title{
Military and Civilian Disease Outbreaks: A Comparative Analysis
}

\author{
Ashlynn Daughton ${ }^{\star 2}$, Nileena Velappan², Esteban Abeyta ${ }^{2,1}$ and Alina Deshpande ${ }^{2}$
}

${ }^{1}$ University of New Mexico, Albuquerque, NM, USA; '2Los Alamos National Laboratory (LANL), Los Alamos, NM, USA

\section{Objective}

Compare and contrast military and civilian outbreaks for malaria and influenza like illness to identify indicators for early warning and detection.

\section{Introduction}

Using influenza like illness (ILI) data from the repository held by AFHSC, and publically available malaria data we characterized similarities and differences between military and civilian outbreaks. Pete Riley et al. utilized a similar ILI dataset to investigate civilian and military outbreaks similarity during the 2009 flu epidemic [1]. They found, overall, high similarity between civilian and military outbreaks, with military peaking roughly one week after civilian. Our analysis is meant to extend their analysis temporally, geographically, and to see if such trends hold true for other diseases.

\section{Methods}

ILI data from January 2000 to December 2014 was obtained from AFHSC. This dataset included any records where the ILI ICD-9 diagnostic code was included in the top 8 diagnostic codes reported by the healthcare facility. Malaria data was collected from peer reviewed literature and official public health reports. Sources included the CDC's MMWR, AFHSC's MSMR, and public health websites.

Military and civilian outbreaks with data from the same location (country or state) and the same time were 'paired'. Outbreaks were compared with respect to time of peak. Additional comparisons including outbreak duration, and comparisons of similarity to laboratory confirmed data, are planned to be completed shortly. All analyses are done using R.

Of note, locations were limited to places where the U.S. military travels that additionally report malaria or ILI. Further, temporal granularity was limited to the highest "timestep" (i.e. if one dataset was reported in months, the 'pair' dataset was aggregated to monthly data as well).

\section{Results}

'Paired' civilian and military malaria and ILI data were available for locations listed in Table 1. Figure one shows data in Afghanistan and South Korea for 2009 to 2012. There is visual similarity between military and civilian data (see Figure 1), but it is not statistically significant. Among the 10 malaria pairs, civilian outbreaks peak between 3 months before and 2 months after military, with an average of 0.1 months after.

We currently have 23 pairs of ILI outbreaks in the United States, Japan and South Korea. Additional data is available and will be analyzed shortly. In the current dataset, there is an average of 5.7 weeks difference in peak, with military peaks typically occurring first. As with malaria, this average has a substantial range $(95 \% \mathrm{CI}$ : 1.8 - 9.5 weeks). Similarity of peak difference depends on location (see Figure 2). In South Korea, civilian outbreaks peak on average 1.6 weeks after military outbreaks ( $95 \%$ CI: $-1.34-4.55)$, while in Japan, civilian outbreaks peak an average of 6.4 weeks after military outbreaks (95\% CI: 1.53 - 11.22).

\section{Conclusions}

This data indicates potential trends among diseases occurring in a particular location, with military outbreaks tending to peak earlier than civilian outbreaks. There is extensive variability between malaria and ILI, as well as variability between locations. Conclusions, especially for malaria, are limited by a lack of granular temporal data and a general lack of military data. Additional data analyses are ongoing to further substantiate these observations and will be completed before the ISDS meeting.

Table 1: Malaria and ILI 'Pairs' and Peak Comparison

\begin{tabular}{|l|c|c|c|}
\hline Disease & $\begin{array}{c}\text { \# Military/ } \\
\text { Civilian } \\
\text { Outbreak }\end{array}$ & $\begin{array}{c}\text { Locations of Military/Civilian } \\
\text { Outbreak }\end{array}$ & $\begin{array}{c}\text { Peak comparison (Civilian - Military) } \\
\text { Average (95\% confidence interval) } \\
\text { (min-max) (units) }\end{array}$ \\
\hline Malaria & 10 & South Korea Afghanistan & $0.11(-0.84,1.06)(-3,2)($ months) \\
\hline Influenza & 23 & $\begin{array}{c}\text { US (North Carolina, California, } \\
\text { Texas) South Korea Japan }\end{array}$ & $5.7(1.8,9.5)(-14,39)($ weeks) \\
\hline
\end{tabular}
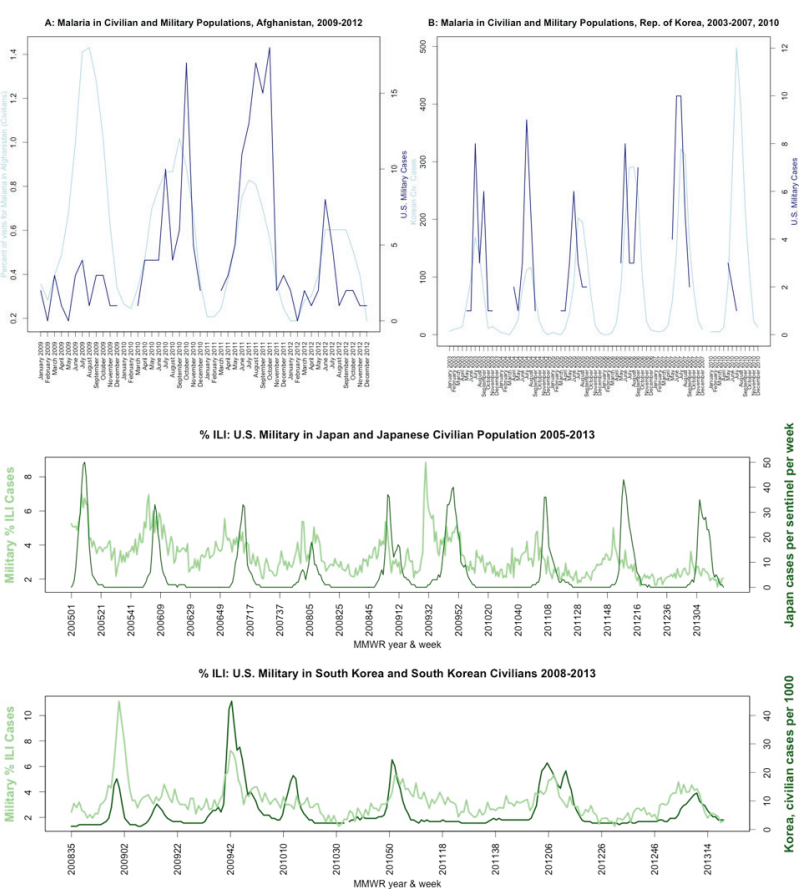

Keywords

Influenza like illness; Malaria; Military; Surveillance

\section{Acknowledgments}

We would like to thank AFHSC for providing us with outbreak data. We would also like to acknowlege Reid Priedhorsky for his computational assistance.

\section{References}

1. Riley P, Ben-Nun M, Armenta R et al. Multiple Estimates of Transmissibility for the 2009 Influenza Pandemic Based on Influenzalike-Illness Data from Small US Military Populations. PLoS Comput Biol. 2013;9(5):e1003064. doi:10.1371/journal.pcbi.1003064.

\section{*Ashlynn Daughton}

E-mail: adaughton@lanl.gov 